\title{
The placebo effect of direct current cardioversion on atrial fibrillation: myth or fact
}

\author{
Authors: Myat Kaung Thu ${ }^{\mathrm{A}}$ and Sanjay Gupta ${ }^{\mathrm{A}}$
}

\section{Introduction}

Atrial fibrillation (AF) is the most common type of arrhythmia across the world, with an estimated prevalence of $2 \%$ in the UK population. ${ }^{1}$ Therapeutic strategies such as medications and AF ablation are used to achieve symptomatic improvement in patients with AF. In our clinical practice, a significant number of patients report symptomatic improvement after direct current (DC) cardioversion. It is as yet unclear as to how much of the purported benefit is due to just a placebo effect. This project aimed to identify the perceived placebo effect of DC cardioversion.

\section{Methods}

A retrospective observational study has been undertaken locally by examining case notes from 100 patients who underwent DC cardioversion from February 2016 to March 2017, which included follow-up data for 24 months.

\section{Results}

Eighty-three AF patients and 17 atrial flutter patients were involved in this project (Table 1). Rate control had been achieved before DC cardioversion in all patients. These patients were prescribed anticoagulant (direct oral anticoagulant (DOAC) or warfarin) for at least 4 weeks before DC cardioversion. AF related symptoms were assessed at the followup clinic following DC cardioversion. Based on the reported symptoms, we collected and analysed symptoms as written in the clinic letter then categorised these into two different groups; improved or not improved. Two patients died and one patient did not attend for follow up. The data for 10 patients did not clearly state the patient symptoms or were not available due to not following up in the clinic letter.

Following DC cardioversion, 48 patients (48\%) achieved sinus rhythm whereas 49 patients $(49 \%)$ remained in arrhythmia (AF or flutter) at the 24-month follow-up point.

In the sinus rhythm group, 38 patients $(79.6 \%)$ reported symptom improvement in relation to the AF-related symptoms. Surprisingly, in the other group, a group of patients who were still reverted to AF, 16 patients (32.65\%) mentioned improved AF-related symptoms following DC cardioversion.
Table 1. Summary of results

Number of Percentage patients

Sinus rhythm $(n=48)$

Improved 38 79.16

Not improved

5

No data

5

10.41

10.41

Atrial fibrillation/flutter $(n=49)$

Improved

16

32.65

Not improved

28

57.14

No data

5

10.20

\section{Conclusions}

Although this study is a subjective and retrospective project, it highlighted that DC cardioversion has a positive impact on patient symptoms, whether rhythm control is achieved or not. By offering DC cardioversion, there will be nearly $50 \%$ chance of getting back to sinus rhythm at the 24-month follow up. It is expected to improve clinical symptoms in four out of five people in whom sinus rhythm is achieved. Even if they revert back to AF or flutter, one in three patients have improved symptoms following DC cardioversion. These findings may be useful in the management of patients suffering from AF-related symptoms who have already achieved rate control.

\section{Conflicts of interest}

None declared.

\section{Reference}

1 Hobbs FD, Fitzmaurice DA. A randomised controlled trial and cost-effectiveness study of systematic screening (targeted and total population screening) versus routine practice for the detection of atrial fibrillation in people aged 65 and over. The SAFE study. Health Technol Assess 2005;9:174. 